\title{
Percepción de los entrenadores sobre la toma de decisión de los jugadores en la iniciación al baloncesto
}

\section{Perception of coaches on decision making of novice basketball players}

\author{
Jiménez, A.C. \\ Universidad Politécnica de Madrid
}

\begin{abstract}
Resumen: El baloncesto es un deporte colectivo de cooperación-oposición. La táctica es un factor importante y está basada en componentes cognitivos y motrices: percepción, decisión y ejecución. Para enseñar este deporte hay alternativas basadas en la táctica. Se trata de comenzar por la comprensión del juego para conseguir los aprendizajes tácticos y técnicos. El presente estudio tiene como objetivo conocer el perfil de un conjunto de entrenadores de baloncesto en la iniciación, respecto a cómo plantean la táctica, en particular el componente de las toma de decisión en la enseńanza. Han participado 89 entrenadores, 72 hombres y 17 mujeres. Han completado un cuestionario con 15 preguntas relacionadas con sus datos personales y sobre el uso del componente táctico en la enseñanza del baloncesto. Los resultados muestran una mayoría de entrenadores jóvenes que fomentan frecuentemente la toma de decisión y utilizan ejercicios de superioridad e igualdad numérica.

Palabras clave: Enseńanza aprendizaje en Baloncesto, entrenadores, tomas de decisión.
\end{abstract}

Abstract: Basketball is a team sport-opposition cooperation. Tactic is an important factor and is based on cognitive and motor components: perception, decision and performance. There are alternatives based on tactics to teach this sport. It is to start by understanding the game to achieve tactical and technical learning. The present study aims to determine the profile of basketball coaches in initiation on how raise the tactic, particularly the component of decision making in teaching. 89 coaches have participated 72 male and 17 female. They have completed a questionnaire with 15 questions related to their personal data and on the use of tactical component in teaching basketball. The results show a majority of young coaches often encourage decision making and use exercises numerical superiority and equality.

Keywords: Teaching and learning in Basketball, coaches, decision making.

\section{Introducción}

La enseñanza del baloncesto en las etapas de iniciación es una de las preocupaciones de los entrenadores y el profesorado implicados en el progreso de los jugadores a quienes enseñan. Así mismo, en el ámbito de la investigación existe una constante inquietud por el proceso de enseñanza-aprendizaje, con la intención de que los resultados obtenidos puedan ser de utilidad y susceptibles de ser llevados a la práctica, tanto en el entrenamiento como en la competición.

Los métodos, modelos y técnicas de enseñanza como medios o recursos a disponibilidad de los entrenadores, pretenden dar respuesta a la cuestión relacionada sobre cómo enseñar, y más concretamente sobre cómo enseñar mejor. Todavía se mantiene vigente, con mayor o menor aceptación, un tipo de clasificación de modelos de enseńanza del deporte en edad escolar denominados modelo tradicional o técnico, y modelo alternativo o centrado en la táctica (Bayer, 1986; Blázquez, 1995; Devís, 1996; López \& Castejón, 2005; Méndez, 1999; 2005). Al modelo técnico se le confieren unas características relacionadas con el empleo de la instrucción directa: la enseñanza analítica basada en la secuenciación de tareas técnicas y repetitivas, dividiendo y jerarquizando los elementos técnicos

específicos; tareas descontextualizadas y con poca o ninguna conexión con las situaciones del juego real. En este modelo, el jugador debe hacer bien una ejecución correcta, aunque queden más al margen las opciones de autonomía y creatividad, así como su capacidad reflexiva en cuanto a las acciones realizadas.

El modelo alternativo o centrado en la táctica surgió como contrapartida al modelo técnico, dado el escaso o nulo protagonismo, en dicho modelo, de una enseńanza que dé prioridad a la mejora de los aspectos cognitivos necesarios para familiarizar al jugador a las situaciones reales del juego (Devís, 1996). La resolución de problemas que plantea el juego, tanto en la fase de ataque como de defensa, manifiesta la necesidad de utilizar los procesos cognitivos de manera eficiente con el fin de lograr los objetivos marcados (Devís, 1996).

Uno de los referentes, en el modelo de enseñanza partiendo de la táctica, es la enseńanza comprensiva del deporte, denominada Teaching Games for Understanding (TGfU) (Bunker y Thorpe, 1982; Oslin \& Mitchell, 2006), que ha tenido un gran calado en el ámbito de la enseñanza del deporte, incluso al margen de la asignatura de Educación Física, aunque este contexto educativo haya sido el punto de partida. Este modelo centra la atención en el jugador, haciéndole partícipe de su propio aprendizaje; utiliza el descubrimiento guiado y la resolución de problemas; plantea una enseñan- 
za global a través de progresiones situacionales que permita plantear tareas contextuales lo más semejantes al juego real. A su vez, propone en la enseñanza el uso de los juegos reducidos, simplificados, o modificados. Favorece el aprendizaje significativo del jugador y una mejora perceptiva y decisional, dando respuesta, no solo a cómo realizar una acción en una situación de juego, sino que prioriza en cuándo, qué y por qué realizar una acción. Otra característica importante se refiere al fomento de la reflexión de los participantes en cuanto a sus decisiones y acciones en la práctica, tanto durante el desarrollo de las sesiones o entrenamientos y competición, como tras su finalización (Castejón, Giménez, Jiménez \& López, 2003; López, 2009; Méndez, 2013).

La comparación de los modelos tradicional y alternativo ha intentado destacar lo más relevante de ambos (Ortega et al., 2015; Turner \& Martinek, 1992; Rovegno, Nevett \& Babiarz, 2001; Rovegno, Nevett, Brock \& Babiarz, 2001). Por otro lado, el énfasis en el estudio de los modelos de enseñanza alternativos ha llevado a diferenciar otros modelos con diversos matices entre sí (Jiménez, 2000). Tal es el caso de los tres modelos comprensivos presentados por López-Ros, Castejón, Bouthier \& Llobet-Martí (2015), en cuanto a la enseñanza comprensiva: (a) TGfU (Bunker \& Thorpe, 1982; Oslin \& Mitchel, 2006); (b) Pedagogía de los modelos de decisión táctica (Gréhaigne, Wallian \& Godbout, 2005); (c) Modelo integrado técnico-táctico (López \& Castejón, 2005).

Abordando la táctica como contenido esencial en el desarrollo del juego de los deportes colectivos de cooperaciónoposición, presentamos una de las diferentes definiciones publicadas sobre la táctica: "Es un proceso de interacción relacionado con parámetros espaciales y temporales donde se muestran comportamientos motrices condicionados por unas normas del deporte, y que tienen como fin conseguir unos resultados que se han definido como principios configuradores de ese mismo deporte." (Castejón \& Argudo, 2012, p. 67). Entendemos que el proceso de interacción implica un proceso cognitivo que permite a los jugadores: (a) reconocer la situación del juego concreta, con todas las variables contextuales del momento; (b) elegir las acciones más apropiadas para obtener el resultado deseado; (c) realizar la acción o acciones técnicas con la suficiente habilidad, coordinación, velocidad y precisión que requiera la situación-problema planteada; y (d) comprobar que lo que se pretendía hacer ha dado resultado.

En el ámbito de la enseńanza del baloncesto, como deporte colectivo de cooperación-oposición, es necesaria la práctica para la mejora de la táctica, bien sea individual o colectiva, de ataque o defensa, incluso en las etapas de iniciación, teniendo en cuenta que el grado de dificultad en la enseñanza táctica evolucionará en función de las características de los jugadores. Entrenar los contenidos tácticos puede ayudar a los jugadores a mejorar sus capacidades perceptivas y decisionales y motrices: (a) focalizando la atención en los estímulos verdadera- mente relevantes; (b) considerando los aspectos relacionados con el espacio (la posición del jugador y las posiciones que ocupan sus compañeros y los adversarios; la posición del jugador con relación a la canasta, etc.); (c) adaptando sus decisiones y acciones con relación al factor tiempo (tiempo de juego, tiempo de posesión); (d) actuando de un modo u otro en función del resultado del partido (aunque esta variable no deba ser el fin primordial en las etapas de iniciación); (e) eligiendo en cada momento la mejor opción de entre las diferentes alternativas que presente una situación concreta del juego; (f) realizando los reajustes necesarios en función de las sucesivas y rápidas situaciones contextuales que presenta el juego; y (g) teniendo en cuenta aspectos estratégicos previamente establecidos.

¿Y, qué importancia tiene la toma de decisiones en baloncesto? La inmensa mayoría de las acciones realizadas por los jugadores durante la competición implica una toma de decisiones. Este es un proceso deliberado de interacción constante entre el jugador y la situación-problema que plantea el juego. Las decisiones y acciones se entremezclan como un continuo ante las múltiples situaciones cambiantes, favoreciendo la adaptabilidad y eficacia del comportamiento los jugadores (Araújo, 2011).

La toma de decisión ha sido y es objeto de estudio en numerosas investigaciones con diferentes perspectivas tales como las teorías cognitivistas, o la perspectiva ecológica. (Araújo \& Passos, 2008; Gibson, 1979; Pozo, 1993; Ruiz \& Arruza, 2005; Tenembaun, 2003). Aquellas teorías o perspectivas en las que se asume que el ser humano tiene capacidad de pensar y decidir, son las que pueden aportar investigaciones que avalan este aspecto (Bonome, 2009; Clark, 1999; Kahneman, 2012).

Existen factores determinantes en la toma de decisiones tales como: las propias capacidades de los jugadores; las tareas que deben resolver, y el contexto en donde se desarrolla la acción (Lago, 2009). Partiendo de estos tres bloques, podemos distinguir determinados elementos que pueden influir en la toma de decisiones de los deportistas: (a) la capacidad perceptiva del deportista, incluyendo en este apartado el comportamiento visual (Abernethy, 1991; Sampedro, Lorenzo \& Refoyo, 2001), la atención (Cárdenas, 2003a), la memoria (Baddeley, 1999), la anticipación; (b) el número de alternativas a elegir; (c) el nivel de riesgo o incertidumbre (este nivel de riesgo puede ser objetivo y subjetivo (Smith \& Kosslyn, 2008); (d) las ideas previas o premisas sobre las que el jugador juzga la situación; (e) el tipo de conocimiento, declarativo y procedimental (Claver, Fernández-Echeverría, Gil, Moreno \& Moreno, 2015; De la Vega, del Valle, Maldonado \& Moreno, 2008; French et al., 1996; Iglesias et al., 2002); (f) el perfil decisional del jugador (García, Ruiz \& Graupera, 2009; Jiménez, Ortega, Giménez \& Castejón, 2015; Jiménez, Sáenz-López, Ibáñez \& Lorenzo, 2012). 
Centrando la atención en las posibles propuestas para la mejora de la táctica en los jugadores de baloncesto, y atendiendo a las variables estructurales del baloncesto: balón, espacio, tiempo, y jugadores, Cárdenas (2009), presenta determinadas orientaciones sobre cómo incrementar las demandas perceptivas y decisionales de los jugadores. De este modo, las tareas en los entrenamientos se plantean alterando, en mayor o menor grado, las reglas del juego establecidas; por ejemplo, jugar con dos balones simultáneamente; reducir el espacio de juego de los atacantes; estipular un tiempo determinado para realizar una acción; aumentar el número de jugadores, o bien el número de alternativas de acción. Basándonos en una distribución de contenidos tácticos, el mismo autor (Cárdenas, 2003b) en el marco de la formación táctica desde una perspectiva constructivista, realiza una clasificación de cinco etapas. Nos vamos a centrar, sintéticamente, en algunos aspectos de las tres primeras etapas, entendiendo que son éstas las que se encuentran más vinculadas a las etapas de iniciación y formación. En la Etapa 1: situaciones de 1x1; medios tácticos colectivos básicos, muy simples, basados sobre todo en las acciones individuales con balón y ocupación de espacios libres; máxima libertad; situación de competición de 3x3. Etapa 2: juego con balón con ayuda, y juego simple con balón; actuación libre y espontánea de los jugadores; fijación directa del impar, bloqueo directo, y pase-desplazamiento; máxima libertad; situación de competición de 3x3. Etapa 3: juego sin balón con ayuda; situaciones de $2 \times 2$, bloqueo directo con intermediario, pase y progresión con intermediario, y el juego en triángulo; juego semilibre; situación de competición de $4 \times 4$.

Otro planteamiento para la progresión del aprendizaje táctico (Junoy, 1996) también presenta tres etapas que están dirigidas a jugadores que se encuentran en edad escolar. En la primera, la enseñanza y aprendizaje del juego individual (recursos en el 1x1; jugar al baloncesto 3x3). La segunda etapa se refiere a la enseñanza y aprendizaje del juego individual con ayuda (recursos en el 1x1+1; iniciación al desmarque; jugar al baloncesto $3 \times 3$ ). En la tercera etapa el autor propone la enseñanza y aprendizaje del juego de asociación (juego de $2 \times 2$ y $3 \times 3$; jugar al baloncesto $3 \times 3$ y $5 \times 5$ ). Es conveniente aclarar que estas propuestas tácticas pretenden la mejora en el aprendizaje táctico de los jugadores, tanto en la fase de ataque como de defensa, aunque esta última fase parezca más difuminada en estas propuestas.

Partiendo del modelo de enseñanza comprensiva, las escuelas sociodeportivas de la Fundación Real Madrid (Ortega, Abad et al., 2015; Ortega, Giménez et al., 2012) fomentan el desarrollo del aprendizaje táctico proponiendo una distribución de contenidos en cada una de las categorías convencionales de baloncesto, desde benjamín hasta cadete, dando por hecho que dichos contenidos deben adaptarse según las características de los jugadores y del equipo. Así mismo, se aporta algún tipo de propuesta u orientación táctica a cada contenido; por ejemplo, recibir el balón: la propuesta sugerida en la categoría benjamín es desarrollar la capacidad de desplazarse y cambiar de ritmo para recibir el balón; en la categoría alevín, fomentar el desmarque en función de la posición del balón y el defensor; en la categoría infantil, seleccionar distintos tipos de cortes con la utilización de fintas y cambios de dirección en función del defensor. Los jugadores deben comprender qué hacer, cómo hacerlo, cuándo hacerlo y hacerlo lo mejor posible. Fomentan los juegos reducidos en situaciones de $1 \times 1,2 \times 1,2 \times 2,3 \times 1,3 \times 2$ y $3 \times 3$ fundamentalmente. La competición en minibasket es un torneo interno de $3 \times 3$ en medio campo, sin presencia de árbitro (hay un orientador que guía a los jugadores en caso de necesidad). En categoría infantil, 4x4 en todo el campo. Tanto en los entrenamientos como en la competición se fomenta la reflexión a través de feedbacks interrogativos, así como a través de tiempos de reflexión al finalizar las actividades.

Los entrenadores en las etapas de iniciación son figuras imprescindibles en el aprendizaje de los jóvenes deportistas. Adquieren una gran responsabilidad, puesto que no solo transmiten sus conocimientos específicos, sino que son claves en la formación integral de sus jugadores desde los ámbitos motor, psicológico y afectivo-social.

La formación inicial y continua de los entrenadores es indispensable para garantizar un proceso de enseńanza y aprendizaje deportivo óptimo, sin fisuras, que favorezca el progreso, la motivación y el bienestar de los jugadores. Es posible que el perfil de los entrenadores de las categorías de iniciación pueda coincidir con unos jóvenes aprendices, en pleno proceso de formación (Abad, 2013; Abad, Giménez, Robles \& Castillo, 2013; Giménez, 2003, Giménez \& Sáenz-López, 2003; Jiménez \& Lorenzo, 2009).

Para entrenar en la iniciación, Giménez (2003) establece tres factores básicos que deben tener en cuenta los entrenadores: conocimiento específico del deporte; conocimiento sobre los jugadores, y conocimiento sobre los fines apropiados para conseguir los objetivos propuestos. Entendemos que este último factor incluye fines pedagógicos que permitan la formación integral de los deportistas. En este sentido, cabe destacar en la actualidad la puesta en acción de proyectos educativos a través del deporte, con un nivel de satisfacción reconocido por todos los agentes implicados (entrenadores, jugadores y familias); un ejemplo de ello son los trabajos realizado por Ortega et al. (2012, 2015).

La percepción que tienen los entrenadores en las etapas de iniciación al baloncesto con relación a cómo y qué enseñan a sus jugadores han sido objeto de estudio. Así mismo, se ha indagado sobre las características personales de los entrenadores y sobre su formación, generando un perfil de estos docentes. Como hemos mencionado anteriormente, algunos estudios coinciden en que los entrenadores de 
iniciación suelen ser jóvenes, con una formación inicial incipiente en su mayoría. Parecen coincidir en la necesidad de formación específica y educativa, y la responsabilidad pedagógica que debe tener el entrenador en la iniciación (Abad et al., 2013; Giménez, 2013; Vizcaíno, Sáenz-López, Rebollo \& Conde, 2014). En el caso concreto de la investigación de Giménez (2013), el perfil de los entrenadores de escuelas de baloncesto revela una escasa presencia de mujeres, y un nivel de experiencia igual o menos de tres años de los entrenadores de minibasket.

En cuanto a la percepción de los entrenadores, respecto a los contenidos tácticos, los estudios analizados indican que los entrenadores los trabajan desde las categorías de minibasket, partiendo de consignas simples y avanzando en progresión según las categorías. En el estudio de Vizcaíno et al. (2014) realizado con entrenadores de minibasket, con relación a la táctica estos plantean la necesidad de utilizar el juego, situaciones reducidas con implicación cognitiva. Otros estudios (Salado, Bazaco, Ortega \& Gómez, 2011) también han analizado la opinión de los entrenadores en cuanto a la distribución de los medios técnico-tácticos, desde categorías de iniciación hasta categoría júnior; centrándonos en las etapas de minibasket e infantil, en cuanto a los medios técnico-tácticos individuales, destacan el bote, el pase y recepción y la defensa al jugador con balón. Respeto a los medios tácticos colectivos básicos, Leite, Gómez, Lorenzo \& Sampaio, (2011), para edades de 10 a 14 años, destacan la presencia de las situaciones reducidas de $2 \times 2$ y $3 \times 3$; situaciones formales del juego; superioridad numérica; y más trabajo de ataque que de defensa. Ortega (2010) y Salado et al. (2011) coinciden en que el medio táctico preferido por los entrenadores en minibasket e infantil es pasar y cortar y a continuación penetrar y doblar. En cuanto a los contenidos tácticos colectivos complejos, el contraataque es el contenido más valorado, seguido del ataque posicional. Ambos estudios coinciden en que los entrenadores plantean una progresión de los medios tácticos que evoluciona de según las diferentes categorías. Atendiendo a todos estos aspectos que se mencionan, en particular a la incidencia del entrenador en la mejora de la toma de decisiones en los jugadores, creemos que todavía necesitamos conocer más sobre cómo se plantea la enseńanza desde la táctica, y por ello es preciso investigar cómo diseñan y ponen en práctica las actividades para mejorar la toma de decisión en baloncesto.

Dada la importancia de los entrenadores en el proceso de enseñanza y aprendizaje táctico, hemos centrado la atención en la percepción que estos tienen sobre la táctica; por tanto, los objetivos propuestos en este estudio han sido: conocer el perfil de los entrenadores de baloncesto de iniciación, y analizar la percepción de dichos entrenadores sobre la toma de decisión y qué actividades plantean para su desarrollo y mejora.

\section{Método}

\section{Participantes}

Los participantes de este estudio han sido 89 entrenadores, de los cuales 17 son mujeres. Pertenecen a clubes y centros educativos de la Comunidad de Madrid. Los criterios para su elección estuvieron basados en que fueran entrenadores o entrenadoras de minibasket o de categoría infantil.

\section{Instrumento}

Para la realización de este estudio se utilizó un cuestionario con 15 preguntas de acuerdo a los objetivos de la investigación. Previamente a la elaboración del instrumento definitivo, éste se propuso a tres expertos para su validación habiéndose utilizado solamente aquellos ítems en los que los expertos estuvieron en total acuerdo. Si algún ítem no era confirmado por alguno de los expertos, se eliminó del cuestionario definitivo. Una vez aportada su valoración y algunos aspectos que debieron modificarse, se procedió al documento definitivo.

\section{Procedimiento}

Este estudio se llevó a cabo con la colaboración de un grupo de estudiantes de una Facultad de Ciencias de la Actividad Física y del Deporte y de los entrenadores participantes, previo consentimiento informado sobre el desarrollo de este estudio y del cuestionario a realizar. Para contar con la colaboración de los entrenadores, se tuvo en cuenta la facilidad de contacto y de acceso para participar en este estudio.

Posteriormente, se les entregó el cuestionario, previa explicación concreta de los objetivos y los motivos del estudio, así como la resolución de alguna duda planteada. Tras la cumplimentación fueron entregados y dispuestos para su posterior análisis.

Se realizó un análisis descriptivo de las respuestas obtenidas basado en frecuencias y porcentajes con la utilización de Excel.10.

En los ítems que se solicitaban breves respuestas abiertas, se analizó el contenido de éstas y se agruparon por temas específicos, como fue el caso de las actividades realizadas para la mejora de las tomas de decisión. En estos casos se intentó identificar el grupo de respuestas comunes con una o dos palabras, o una frase corta representativa respecto al sentir de los entrenadores, por ejemplo "saber leer el juego, percibir”; a partir de ahí, se cuantificó el número de veces que los entrenadores expresaron este término, y así con el resto de respuestas. La intencionalidad fue presentar la frecuencia con la que los entrenadores que participaron se inclinaban por unos términos u otros. 


\section{Resultados}

En las tablas 1 y 2 se muestran los resultados de los entrenadores en cuanto a género, edad, nivel de experiencia, título federativo que poseen, categoría del equipo en el que entrena y centro o club donde entrenan. Las tablas 3 y 4 presentan los resultados respecto a la precepción de los entrenadores sobre la toma de decisión y propuestas de actividades para su mejora.

\section{Perfil de entrenador}

Tabla 1. Perfil del entrenador con datos relacionados con el género edad y ańos de experiencia.

\begin{tabular}{lcccccccc}
\hline Género & $\mathrm{F}$ & $\%$ & Edad & $\mathrm{F}$ & $\%$ & Experiencia & $\mathrm{F}$ & $\%$ \\
\hline Masculino & 72 & 81 & $20-25$ & 60 & 67.4 & Menos 3 años & 36 & 40.5 \\
Femenino & 17 & 19 & $26-30$ & 9 & 10.1 & Entre 3 y 8 años & 35 & 39.3 \\
\hline
\end{tabular}

Tabla 2. Perfil del entrenador con datos relacionados con su titulación federativa, categoría y entidad en donde entrenan.

\begin{tabular}{|c|c|c|c|c|c|c|c|c|}
\hline Titulación federativa & $\begin{array}{c}\mathrm{N}=89 \\
\mathrm{~F}\end{array}$ & $\%$ & Categoría & $\begin{array}{c}N=89 \\
F\end{array}$ & $\%$ & Entidad & $\begin{array}{c}N=55 \\
F\end{array}$ & $\%$ \\
\hline Ninguna & 16 & 18 & Benjamín & 26 & 29.2 & Colegios & 17 & 30.9 \\
\hline Nivel 0.0 & 26 & 29.2 & Alevín & 27 & 30.3 & Asoc. Deport & 7 & 12.7 \\
\hline Nivel 1 & 32 & 36 & Infantil & 36 & 40.5 & Clubes & 31 & 56.4 \\
\hline Nivel 2 & 12 & 13.5 & & & & & & \\
\hline Entrn. superior & 3 & 3.3 & & & & & & \\
\hline
\end{tabular}

Los entrenadores participantes, son mayoritariamente hombres (72). También hay mayoría de entrenadores jóvenes con edades comprendidas entre 20 y 25 ańos; a continuación le sigue el grupo de más de 30 años, y por último le sigue la franja de edad entre 26 y 30 años. Respecto a los ańos de experiencia, los resultados son similares entre los que han entrenado menos de tres ańos y los que llevan entre tres y ocho años. Es claramente menor el número de entrenadores que supera los ocho años de experiencia como entrenador.

En cuanto a la formación específica de baloncesto valorada a través del título federativo de los entrenadores, la mayoría de ellos tienen el nivel 1; a continuación el nivel 0.0; le sigue los entrenadores que no tienen ninguna titulación, y los que tienen el nivel 2. El número de entrenadores con el título de entrenador superior es ínfimo. Respecto a las categorías a las que entrenan, es mayor la categoría infantil; a continuación, con valores muy similares la categoría alevín y benjamín. En cuanto a la entidad en donde entrenan, se dio la circunstancia de que algunos entrenadores coincidieron en entrenar en la misma entidad, pero con distintos equipos, de ahí, que solo fueron 55 entidades. En primer lugar se encuentran los clubes de baloncesto; a continuación los centros escolares y en menor porcentaje las asociaciones deportivas municipales.
Tabla 3. Percepción del entrenador sobre la toma de decisión de los jugadores.

\begin{tabular}{lcc}
\hline $\begin{array}{l}\text { ¿Qué es para ti la toma de decisiones en jugadores } \\
\text { de baloncesto? }\end{array}$ & $\mathrm{N}=89$ & $\%$ \\
\hline $\begin{array}{l}\text { Saber elegir o capacidad de elegir y resolver. Saber } \\
\text { qué hacer }\end{array}$ & 22 & 24.7 \\
\hline Autonomía. Libertad & 10 & 11.2 \\
\hline $\begin{array}{l}\text { Saber leer el juego. Percibir estímulo y decidir. } \\
\text { Pensar y elegir }\end{array}$ & 9 & 10.1 \\
\hline Herramienta para crecer & 4 & 4.5 \\
\hline Aprender de los errores & 4 & 4.5 \\
\hline Muy importante. Fundamental. Esencial. & 33 & 37.1 \\
\hline No es importante /no contesta & 5 & 5.6 \\
\hline
\end{tabular}

Ante la cuestión realizada sobre qué era para los entrenadores la toma de decisión, la respuesta más frecuente fue que era muy importante y esencial. A continuación, se inclinaron por dar nociones conceptuales de la toma de decisión. Con menor frecuencia hubo entrenadores que definieron la toma de decisión como referente de autonomía y libertad; parecido número de entrenadores incluyeron la percepción como factor importante en la toma de decisión. Un número menor de entrenadores, cuatro, destacaron que era una herramienta para crecer, y otros cuatro lo relacionaron con la posibilidad de aprender de los errores. Hubo cinco entrenadores a los que no les pareció importante, o no dejaron constancia alguna de su parecer. 
Tabla 4. Actividades realizadas por los entrenadores para la mejora de la toma de decisión.

\begin{tabular}{lccc}
\hline $\begin{array}{l}\text { Ejemplos de actividades para el fomento de la toma de } \\
\text { decisión en baloncesto }\end{array}$ & F & $\%$ \\
\hline Solo $1 \times 1$ & 12 & 13.5 \\
Situaciones de igualdad 1x1, 2x2 & 15 & 16.8 \\
Situaciones de superioridad numérica. 2x1, 3x2 & 13 & 14.6 \\
Resolución de problemas; dar más de una opción & 17 & 19.1 \\
$3 \times 3,4 \times 4$ & 7 & 7.8 \\
Situaciones reales de partido. 5x5 & 9 & 10.1 \\
Ante el error fomentar la reflexión de por qué & 5 & 5.6 \\
Generar TD muy rápidas & 3 & 3.4 \\
TD con condicionantes o muy ventajosos & 2 & 2.2 \\
Variabilidad de ejercicios & 3 & 3.4 \\
Al finalizar la tarea preguntar el porqué de esa opción. & 2 & 2.2 \\
Al finalizar la tarea dar información & 2 & 2.2 \\
Leer el juego, pensar, comprender el juego & 4 & 5 \\
Otras situaciones concretas de un partido; por & 3 & 3.4 \\
ejemplo: cuando el equipo pierde de 10 a falta de un & & \\
cuarto, incita a que busquen soluciones para remon- & & \\
tar/Refuerzos positivos-negativos/Visionado de vídeos. & & \\
Pocas veces trabajan las tomas de decisión & 3 & 3.4 \\
Nunca trabajan las tomas de decisión & 1 & 1.1 \\
\hline
\end{tabular}

Los resultados mostrados en esta tabla indican las actividades más frecuentes realizadas por los entrenadores. Cabe destacar que algunos de ellos indicaron más de una actividad. El orden de las tareas más frecuentemente realizadas fueron: la resolución de problemas; a continuación situaciones de igualdad 1x1 y 2x2; seguido por situaciones de ataque en superioridad numérica $2 \times 1$ y 3x2; le siguió los ejercicios de $1 \mathrm{x} 1 \mathrm{y}$, posteriormente, las situaciones reales de juego, 5x5. La opción de ofrecer información al finalizar la tarea, así como ofreciendo un feedback interrogativo fueron las menos recurridas por los entrenadores. Sólo cuatro entrenadores indicaron que trabajan pocas veces, o nunca, las tomas de decisión.

\section{Discusión}

Los resultados obtenidos, relacionados con el perfil de los entrenadores participantes en el presente estudio, reflejan un prototipo parecido al de otras investigaciones en cuanto a que, en su mayoría, son jóvenes entrenadores los que enseñan en las etapas de minibasket, y dada esta juventud, se caracterizan por tener generalmente un nivel de experiencia bajo (Abad, 2013; Abad et al., 2013; Giménez, 2003; Giménez \& Sáenz-López, 2003). Esta evidencia revela la necesidad de una formación inicial y permanente de calidad, tanto de carácter genérico como específico. De ahí la importancia de los centros de formación, bien sea universitaria o federativa, para dotar a los entrenadores de las competencias específcas de baloncesto, así como pedagógicas, que permitan a los entrenadores contribuir con su enseñanza a la formación integral de sus jugadores (Carreras et al., 2009; Giménez, 2003; Ortega, Giménez et al., 2012). En este estudio hemos detectado que existen pocos entrenadores con más de ocho años de experiencia $\mathrm{y}$, a su vez, son pocos entrenadores los que tienen una titulación federativa de nivel 2 o entrenador superior; consideramos que si entrenaran en categorías superiores, posiblemente ambos valores fueran más altos (Jiménez \& Lorenzo, 2009). Otro aspecto que hemos constatado, es la escasa presencia de mujeres como entrenadoras, desconociendo el porqué de este hecho; en este sentido coincide con la investigación de Giménez (2013), aunque no hallamos respuesta a esta poca presencia. Por otro lado, en cuanto a la entidad en donde entrenan, pertenecientes a la Comunidad de Madrid, de los 55 centros constatados, la mayoría son clubes de baloncesto, algunos de ellos con una dilatada trayectoria no sólo como formadores de la cantera, sino con equipos punteros en alta competición. Cabe destacar que casi un tercio de los centros a los que pertenecen los entrenadores son colegios, algunos de ellos también pioneros en la formación de jóvenes jugadores. Por último, se encuentran las asociaciones deportivas municipales.

En cuanto a la percepción de los entrenadores sobre las tomas de decisión de los jugadores, ante la pregunta recogida en la tabla 3 "¿qué es para ti la toma de decisión en jugadores de baloncesto?” dicho ítem fue lo suficiente amplio como para que hubiera diferentes respuestas; algunas orientadas hacia una breve definición de la toma de decisión; otras indicaron concisamente que las tomas de decisión eran muy importantes; algunos entrenadores optaron por la inclusión de un único término que definiera la toma de decisión, como libertad o autonomía; otros entrenadores se inclinaron por incidir en los procesos de percepción y decisión. Consideramos que estas apreciaciones, desde la perspectiva del conocimiento declarativo de los entrenadores, aportan matices bastante acertados (Iglesias et al., 2002; Ruiz \& Arruza, 2005). También destacamos que para una minoría de entrenadores la toma de decisión es un instrumento para mejorar; para ellos es un medio para "aprender de los errores", entendemos que se refieren a cuando se toman decisiones erróneas; también es una "herramienta para crecer”. Por último, indicar que una minoría no tuvieron en consideración las tomas de decisión; es posible que fueran entrenadores más afines con el modelo técnico, en donde prevalece más la enseńanza analítica (Méndez, 1999, 2005).

Respecto a las actividades planteadas por los entrenadores con el fin de mejorar la toma de decisiones de sus jugadores, las respuestas de los entrenadores se encuentran en consonancia con los contenidos propuestos en otras publicaciones 
para la mejora del aprendizaje táctico (Cárdenas, 2003b; Junoy, 1996; Ortega, Abad et al., 2015; Ortega, Giménez et al., 2012). Las diferentes alternativas de juego reducido con situaciones de igualdad $1 \mathrm{x} 1$ y $2 \mathrm{x} 2$ y superioridad numérica, $2 \times 1$ y $3 \times 2$, coinciden también con otros estudios, como el de Vizcaíno et al. (2015). Cabe destacar que un grupo de entrenadores focalizaron la atención estrictamente en la propuesta de $1 x 1$; en este sentido nos parecería más adecuado utilizar situaciones de juego con la presencia de al menos un compañero en la fase de ataque, pues aunque se planteen tareas simples, es conveniente mejorar el aprendizaje de los contenidos tácticos colectivos de ataque y defensa, incluso en categorías de iniciación (Cárdenas, 2003b). En línea con los aspectos mencionados la opción de "resolución de problemas dando varias opciones posibles" fue destacada entre los entrenadores; también hubo respuestas sobre la necesidad de "leer el juego" un aspecto que consideramos directamente relacionado con la capacidad perceptiva. Así mismo, algunos entrenadores se decantaron por situaciones de 3x3, 4x4 y $5 \times 5$. Dichas respuestas dan sentido a las situaciones de juego reducido, así como a las situaciones semejantes al juego real que, bien estructuradas, por parte del entrenador pueden generar múltiples y correctas tomas de decisión en los jugadores, y por tanto un apropiado aprendizaje táctico. (Leite et al., 2011; Ortega, 2010; Salado et al., 2011). También estuvieron presentes respuestas relacio- nadas con la variabilidad de los ejercicios, generar tomas de decisión rápidas, ser capaz de decidir correctamente cuando el resultado es adverso en la competición; estos son aspectos que son considerados como variables contextuales que pueden influir a la toma de decisiones y hay que tener en cuenta (Lago, 2009). Sólo una minoría de entrenadores se decantó por aportar conocimiento de resultados, dando información a los jugadores sobre cómo hacerlo mejor, o bien de carácter interrogativo; nos inclinamos por este tipo de feedback considerando que favorece en los jugadores mayor capacidad de reflexión y comprensión del juego. Por último, con relación a los pocos entrenadores que no plantearon tareas que implicaran la toma de decisión, como hemos mencionado anteriormente, tal vez sea por una tendencia hacia el modelo de enseñanza técnico.

Este estudio presenta ciertas limitaciones, tales como una posible mejora en el instrumento utilizado, incrementando y matizando algunos de los ítems. Asimismo, podría ser de interés correlacionar algunas de las variables presentadas. Por otro lado, proponemos estudios futuros en donde se puedan complementar el análisis de la percepción de los entrenadores sobre las tomas de decisión, con el análisis de las actividades prácticas en la cancha, que realizan con sus equipos tanto en los entrenamientos como en la competición.

\section{Referencias}

1. Abad, M.T. (2013). La formación del entrenador en el proceso de enseñanza-aprendizaje de jóvenes futbolistas. En F.J Castejón, F.J. Giménez, F. Jiménez \& López, V. (Eds.), Investigaciones en formación deportiva. (pp. 443-469). Sevilla: Wanceulen.

2. Abad, M.T., Giménez, F.J., Robles, J. \& Castillo, E. (2013). La formación de los entrenadores de jóvenes deportistas. E-Balonmano.com: revista de Ciencias del Deporte, 9(2), 105-114.

3. Abernethy, B. (1991). Visual search strategies and decision-making in sport. International Journal of Sport Psychology, 22, 189-210.

4. Araújo, D. (2011). De la toma de decisiones, al curso de las decisiones. Revista de Psicología del Deporte, 20(2), 639-643.

5. Araújo, D. \& Passos, P. (2008). Fundamentos do treino da tomada de decisao em desportos colectivos com bola. En F. Tavares, A. Graça, J. Garganta \& I. Mesquita (Eds.), Olhares e contextos da performance nos jogos desportivos. (pp. 70-78). Oporto: Faculdade de Desporto.

6. Baddeley, A. (1999). Memoria humana. Teoría y práctica. Madrid: McGraw-Hill.

7. Bayer, C. (1986). La enseñanza de los juegos deportivos colectivos. Barcelona: Hispano Europea.

8. Blázquez, D. (1995). Métodos de enseńanza de la práctica deportiva. En D. Blázquez (Ed.), La iniciación deportiva y el deporte escolar (pp. 251286). Barcelona: Inde.

9. Bonome, M.G. (2009). La racionalidad en la toma de decisiones: Análisis de la Teoría de la Decisión de Herbert A. Simon. La Coruña: Netbiblio.

10. Bunker, D. \& Thorpe, R. (1982). A model for the teaching of games in secondary schools. Bulletin of Physical Education, 18(1), 40-44.

11. Cárdenas, D. (2003a). El entrenamiento perceptivo en baloncesto. En J. Sampedro, A. Lorenzo, C. Jiménez \& I. Refoyo (Eds.), III Curso de preparación física en baloncesto de formación y alto nivel (pp. 1-38). Madrid: CSD.

12. Cárdenas, D. (2003b). El proceso de formación táctica colectiva desde una perspectiva constructivista. En A. López, C. Jiménez \& R. Aguado (Eds.), Curso de Didáctica del Baloncesto en las Etapas de iniciación. (pp. 179-209). Madrid: Autoras.

13. Cárdenas, D. (2009). Bases para la construcción de una progresión didáctica. En G. Ortega \& A.C. Jiménez, (Eds.), Táctica y técnica en la iniciación al baloncesto. (pp. 197-215). Sevilla: Wanceulen.

14. Carreras, Ll., Eijo, P., Estany, A. Gómez, M.T., Guich, R., Mir, V.,... Serrats, M.G. (2009). Cómo educar en valores (15a ed.). Madrid: Narcea.

15. Castejón, F.J., Giménez, F.J., Jiménez, F. \& López, V. (2003). Concepción de la enseńanza comprensiva del deporte: modelos, tendencias y propuestas. En Castejón, F.J. (Ed.), Iniciación deportiva. La enseñanza y el aprendizaje comprensivo en el deporte (pp. 17-34). Sevilla: Wanceulen.

16. Castejón, F.J. \& Argudo, F. M. (2012). Fundamentos de la estrategia y la táctica deportiva. Madrid: Cultivalibros.

17. Clark, A. (1999). Cerebro, cuerpo y mundo en la nueva ciencia cognitiva. Barcelona: Paidós.

18. Claver, F., Fernández-Echevarría, C., Gil, A., Moreno, A. \& Moreno, M.P. (2015). El conocimiento procedimental en jugadores de voleibol de categoría infantil. SportTK: Revista Euroamericana de Ciencias del Deporte, 4(1), 41-48.

19. De la Vega, R., del Valle, S., Maldonado, A. \& Moreno, A. (2008). Pensamiento y acción en el deporte. Sevilla: Wanceulen.

20. Devís, J. (1996). Educación Física, deporte y currículum. Madrid: Visor.

21. French, K. E., Nevett, M. E., Spurgeon, J. H., Graham, K. C., Rink, J. E. \& McPherson, S. L. (1996). Knowledge representation and problem 
solution in expert and novice youth baseball players. Research Quarterly for Exercise and Sport, 67(4), 386-395.

22. García, V., Ruiz, L.M. \& Graupera, J.L. (2009). Perfiles decisionales de jugadores y jugadoras de voleibol de diferente nivel de pericia. Revista Internacional de Ciencias del Deporte, 14, 123-137.

23. Gibson, J.J. (1979). The ecological approach to visual perception. Hillsdale: Lawrence Erlbaum.

24. Giménez, F.J. (2003). La formación del entrenador en la iniciación al baloncesto. Sevilla: Wanceulen.

25. Giménez, F.J. (2013). La formación del entrenador de iniciación al baloncesto en Andalucía. Seminario de formación y estudio de casos. En F.J Castejón, F.J. Giménez, F. Jiménez \& López, V. (Eds.). Investigaciones en formación deportiva. (pp. 327-342). Sevilla: Wanceulen.

26. Giménez, F.J. \& Sáenz-López, P. (2003). El entrenador de Baloncesto en las etapas de formación. En A. López, C. Jiménez \& R. Aguado (Eds.), Curso de Didáctica del Baloncesto en las Etapas de iniciación. (pp. 179-209). Madrid: Autoras.

27. Gréhaigne, J.F., Wallian, N. \& Godbout, P. (2005). Tactical-decision learning model and students' practices. Physical Education and Sport Pedagogy, 10(3), 255-269.

28. Iglesias, D., Moreno, P., Ramos, L. A., Fuentes, J. P., Julián, J. A. \& Del Villar, F. (2002). Un modelo para el análisis de los procesos cognitivos implicados en la toma de decisiones en deportes colectivos. Revista de Entrenamiento Deportivo, 16(15), 9-14.

29. Jiménez, A. C., Ortega, G., Giménez, J. F. \& Castejón, F.J. (2015). Making profiles in boys and girls playing basketball. Revista de Psicologia del Deporte, 24(supl. 1), 51-53.

30. Jiménez, A.C., Sáenz-López, P., Ibáńez, S.J., \& Lorenzo, A. (2012). Percepción de las jugadoras internacionales de baloncesto sobre su toma de decisiones. Revista Internacional de Medicina y Ciencias de la Actividad Física y el Deporte, 12(47), 589-609.

31. Jiménez, F. (2000). Estudio praxiológico de la estructura de las situaciones de enseñanza en los deportes de cooperaciónloposición de espacio común y participación simultánea: balonmano y fútbol sala. Gran Canaria: Universidad de Gran Canaria. Tesis doctoral no publicada.

32. Jimenez, S. \& Lorenzo, A. (2009). La formación de los entrenadores de baloncesto. Aplicaciones didácticas. Sevilla: Wanceulen.

33. Junoy, J. (1996). Baloncesto. Madrid: MEC.

34. Kahneman, D. (2012). Pensar rápido, pensar despacio. Barcelona: Debate.

35. Lago., C (2010). La toma de decisión desde la perspectiva de los sistemas complejos. La influencia de las variables contextuales de la competición en el comportamiento de los jugadores en los deportes de equipo. En V. López \& J. Sargatal (Eds.), La táctica deportiva y la toma de decisiones (pp. 105-137). Girona: Universitat de Girona.

36. Leite, N., Gómez, M., Lorenzo, A. \& Sampaio, J. (2011). Los contenidos de entrenamiento en baloncesto en función de las etapas de preparación deportiva a largo plazo. Revista de Psicología del Deporte, 20(2), 287-303.

37. López, C. (2009). Iniciación al baloncesto. Consideraciones para una enseñanza eficaz. Barcelona: Inde.

38. López, V. \& Castejón, F.J. (2005). La enseñanza integrada técnico-táctica de los deportes en edad escolar. Explicación y bases de un modelo. Apunts. Educación Física y Deportes, 79, 40-48.
39. López-Ros, V., Castejón, F.J., Bouthier, D. \& Llobet-Martí, B. (2015). Modelos para una enseñanza comprensiva del deporte. Espacios comunes para el encuentro (y algún desencuentro). Ágora par la Educación Física y el Deporte, 17(1), 45-60.

40. Méndez, A. (1999). Modelos de enseńanza deportiva. Análisis de dos décadas de investigación. Lecturas: Educación Física y Deportes, 13. www.efdeportes.com

41. Méndez, A. (2005). Técnicas de enseñanza en la iniciación al baloncesto. Barcelona: Inde.

42. Méndez, A. (2013). Estudio comparado sobre la enseñanza del baloncesto en el contexto de Educación Física. En F.J. Castejón, F.J. Giménez, F. Jiménez \& V. López (Eds.), Iniciación deportiva. La enseñanza y el aprendizaje comprensivo en el deporte (pp. 65-90). Sevilla: Wanceulen.

43. Ortega, E. (2010). Medios técnico-tácticos colectivos en baloncesto en categorías de formación. Revista Internacional de Medicina y Ciencias de la Actividad fisica y el Deporte, 10(38), 234-244.

44. Ortega, G., Abad, M.T., Durán, L.J., Franco, J., Giménez, F. J., Jiménez, P. J., \& Jiménez, A. C. (2015). Entrenando Fútbol: enseñando Valores. Madrid: Ediciones Gráficas Real Madrid.

45. Ortega, G., Giménez, F.J., Jiménez, A.C., Franco, J., Durán, L.J., \& Jiménez, P.J. (2012). Iniciación al Baloncesto. Madrid: Ediciones Gráficas Real Madrid.

46. Oslin, J. \& Mitchell, S. (2006). Game-centred approaches to teaching physical education. En D. Kirk, D. Macdonald, \& M. O'Sullivan, M. (Eds.), The Handbook of Physical Education (pp. 627-650). Londres: Sage.

47. Pozo, J. I. (1993). Teorías cognitivas del aprendizaje (2a ed.). Madrid: Morata.

48. Rovegno, I., Nevett, M., \& Babiarz, M. (2001). Learning and teaching invasion-game tactics in 4th grade: Introduction and theoretical perspective. Journal of Teaching in Physical Education, 20, 341-351.

49. Rovegno, I., Nevett, M., Brock, S., \& Babiarz, M. (2001). Teaching and learning basic invasion-game tactics in 4th grade: A descriptive study from situated and constraints theoretical perspectives. Journal of Teaching in Physical Education, 20, 370-388.

50. Ruiz, L.M. \& Arruza, J. (2005). El proceso de toma de decisiones en el deporte. Clave de la eficiencia y el rendimiento óptimo. Barcelona: Paidós.

51. Salado, J., Bazaco, M.J., Ortega, E. \& Gómez, M.A. (2011). Opinión de los entrenadores sobre la distribución de contenidos técnico-tácticos y pedagógicos en distintas categoría de baloncesto de formación. Cuadernos de Psicología del Deporte, 11(2), 51-62.

52. Sampedro, J., Lorenzo, A. \& Refoyo, I. (2001). Percepción en baloncesto. Test de habilidades visuales. En S.J. Ibáñez \& M. Macías (Eds.), Aportaciones al proceso de enseñanza y entrenamiento de baloncesto (pp. 203-209). Cáceres: Autores.

53. Smith, E.E. \& Kosslyn, E. M. (2008). Procesos cognitivos. Modelos y bases neuronales. Madrid: Pearson.

54. Tenenbaum, G. (2003). Expert Athletes: An integrated approach to decision making. En J. L. Starkes \& K. A. Ericsson (Eds.), Expert Performance in sport. (pp. 191-218). Champaign: Human kinetics.

55. Turner, A. P., \& Martinek, T. J. (1992). A comparative analysis of two models for teaching games (technique approach and game centered (tactical focus) approach). International Journal of Physical Education, 29(4), 15-31. 